\title{
Exploring Weak Signals and Wild Cards in the Water Services
}

\author{
Annina Takala ${ }^{a *}$, Ossi Heino $^{a}$ \\ ${ }^{a}$ Department of Chemistry and Bioengineering, Tampere University of Technology, Finland
}

\begin{abstract}
This paper describes a study that was conducted to assess weak signals and wild cards in the case of water services. It is argued that the potential of this approach lies in the practical application, not in scientific strict methodology and rigid interpretations of what weak signals and wild cards are. Thinking about weak signals and wild cards can be an essential part in strategic long-term thinking, help think differently, and in this way contribute to the future sustainability of water services.
\end{abstract}

Keywords: Water Services, Weak Signals, Wild Cards, Strategic Planning.

\section{Introduction}

A variety of futures research methodologies exist and are constantly being developed to scan the environment and to make systems more "sensitive to emerging changes as early as possible so that they have better time to react or to be in time to utilize the opportunities of an emerging change" $[1$, p. 3]. The potential of wild cards and especially weak signals in identifying possible changes in the future that could jeopardise or promote a system's existence have been eagerly discussed and scrutinised [e.g. 2 - 6].

In this paper we examine weak signals and wild cards in the case of water supply and sanitation services ${ }^{1}$. Weak signals and wild cards have typically been analysed in the context of corporate decision-making. Water services provide in many ways an interesting and differing case. For example, water utilities function as monopolies in their operational area, and thus in their case weak signals and wild cards are not about finding a competitive edge in the markets, but about sustaining and ensuring safe services.

In industrialised nations, water services are typically considered highly reliable. People rely on the continuity of these services; they expect to get safe water simply by turning the tap on and wastewater to disappear without causing any trouble to the environment or human health just by flushing the toilet. However, if something goes wrong, this can compromise

${ }^{1}$ We will use the term "water services" to cover both water supply and sanitation services from here onwards. significantly the well-being of many. One example is the water crisis in the Finnish town of Nokia in the year 2007: some 6000 people were taken ill because treated wastewater was accidentally released into the drinking water distribution system. Thus, in the case of water services the question is not just about business opportunities or threats if a wild card materialises, but about human lives and functioning of societies. Water services support either directly or indirectly all socio-economic activities; during the Nokia water crisis, for example, schools and day care centres had to be closed down and many businesses struggled. On the whole, water crisis had long-lasting socio-economic effect for local businesses.

The water services system can also be described a relatively static system. In industrialised countries these systems have mostly been built between the Second World War and the 1980s. During this era development was mostly expected to be linear. For example, many of the pipelines were scaled for stable growth of consumption, which proved unrealistic after the oil crisis in 1970s and the introduction of new water saving household appliances. The infrastructure system, however, is rather inflexible. In essence, there has not been significant changes in the systems after the adaptation of centralised drinking water distribution or water based sanitation. There have been improvements to the treatment technologies of both raw water and wastewater, and the materials and technologies used in the piping systems have developed but in the end, these changes have not really affected the big picture or the paradigm of water services.

\footnotetext{
${ }^{*}$ Corresponding author

E-mail: annina.takala@tut.fi

(C) 2015 International Association for Sharing Knowledge and Sustainability

DOI: $10.5383 /$ swes.7.02.009
} 
Overall, the whole system is relatively inflexible and slow to adapt to changing conditions.

The static nature has contributed to the fact that the sector is rather conservative: somewhat resistant to change and slow to embrace innovations. One reason for this can be related to the fact that water services are considered a natural monopoly. As there has been no need to compete there has been less need for revolutionary changes and innovations in the field. Another reason could be due to the fact that water services are an engineer-oriented sector. As Nafday [7] argues, engineers are generally dismissive of unpredictable events until they occur because engineers are used to focus on specifics and feel uncomfortable with uncertainty and ambiguity. However, these characteristics are not limited to engineers alone but can be generalised to all of us. According to Kahneman [8], people do not cope well with uncertainties but want to believe that by understanding the past it is possible to predict and control the future. In addition, Taleb [9] argues that humans in general focus too narrowly on one's own field of expertise and overestimate one's own knowledge. People tend to underestimate the role of chance and the implications of big changes and overestimate their own capacity to cope with them. To sum up, it is not inherent to organisations in the water services sector - especially those that rely on the bureaucratic organisational culture and construction of the large technical systems - to be adaptive and responsive to changes.

However, the world has changed and also the operational environment of water services is now different. For example, various societal changes, such as the development of the information society, have affected the practices of people and organisations. Nowadays, almost everyone has an access to inexpensive information and communication channels which, in turn, set new standards for the transparency and openness of organisations. From the point of view of water services, this can be seen either as an opportunity or even a threat. It makes organisations more vulnerable if their actions do not meet public expectations but, on the other hand, it can be utilised as a way to better observe and reflect their operational environment.

On the whole, it is debatable whether the water services sector can maintain its static character. There are several challenges facing the water services sector compromising its sustainability [10]. In addition to the identified challenges, there might be some less visible but major changes looming around the corner that could seriously impact the field. It has been assessed that future uncertainty is increasing in the infrastructure sector [11]. Furthermore, due to the nature of water services, there are long delays in the feedback loops. Meadows [12] argues that in such cases foresight is essential; if one acts only when a problem is obvious, then one misses a crucial opportunity to solve the problem.

One of our personal motivations to conduct this study was to give a wakeup call to the field of water services. In our opinion, it needs to be better acknowledged that it is inadequate to examine water services only from the technical perspective and in isolation from the rest of the society. Water services as a system is entangled with and bound within wider sociotechnical, political, cultural and economic complexes [see e.g. 13, 14]. We, in the field of water services, need to be more sensitive to what is happening elsewhere in society and take this into consideration in strategic planning. As Hiltunen [1] argues weak signals can help us to break the prevailing mental models, encourage us to think differently, and help us to be more innovative about the futures.

\section{Materials and Methods}

This section first of all covers a short overview of theory on weak signals and wild cards, and methodology related to them. In the end, the material choice of this study is introduced. The theory in relation to this study is covered in more detail under the Discussion section.

\subsection{Weak Signals}

Igor Ansoff can be considered as the pioneer of weak signals analysis [3]. He defines weak signals as warnings that are too incomplete to permit an accurate estimation of their impact, and/or to determine a complete response [4]. Based on the literature, however, it is not quite obvious what weak signals are but there are many views that are sometimes even contradictory (see e.g. [3] and [15] for an overview). Some use terms 'emerging issues', 'seeds of change', 'wild cards' and 'early warning signals' interchangeably with weak signals [1]. According to Moijanen [15], weak signals are generally defined in three ways: First, some consider that weak signal itself is a changing phenomenon that will strengthen in the future. Second, some see that weak signals are the cause of new phenomena and changes. Third, some limit weak signals as symptoms or signs that indicate change in the future.

Another issue causing confusion in defining weak signals is the debate on their objectivity versus subjectivity; or the essentialist or deterministic versus the constructivist perspective [see 2]. According to the objective view, weak signals exist as such and are independent of the interpreter. Then again, according to the subjective view, weak signal always needs a recipient who interprets the signal [15]. Rossel [16, p. 312] criticises existing literature on weak signals for "neutralising" weak signals, "as if they were objects or features in their own right, waiting to be discovered, instead of considering them as the expression of the paradigmatic capacity of the analyst to organise perception and interpretation in a certain way". Following the subjective view, interpretation of weak signals depends on the context and is situated [4]. Thus, same signal can in one case be interpreted as weak and in another as strong.

Hiltunen [17, p. 249-250] suggests a three-dimensional model of the future sign to clarify the idea of weak signals. The three dimensions are a) the object (emerging issue), b) the representamen (the concrete form that the sign takes, i.e. signal), c) the interpretant (sense made of future potential of the sign). Furthermore, Hiltunen argues that two-dimensions of the sign are objective: object and representamen. These exist as such. Only subjective dimension is interpretation of the sign, as the interpreters make their conclusions about the sign in their own context. [17, p. 249-253] Rossel [5], however, has criticised Hiltunen of overlooking the constructivist approach to weak signals.

\subsection{Wild Cards}

Wild cards have been part of futures research and scenario building since the 1960s. However, it was only in 1996 that an approach to study wild cards came about as John L. Petersen's book Out of the Blue: How to Anticipate Big Future Surprises was published. [18]. Wild cards are generally defined as rapid, surprising events with huge disastrous, destructive, catastrophic or anomalous consequences. Usually these events take place so rapidly that normal, planned management processes cannot respond to them, making the organisations highly vulnerable. 
$[19,20]$ Petersen and Steinmüller [18] argue that in the complex and interconnected world of today, it is now more relevant than ever to study wild cards so that based on the information we could prepare for them, prevent them or in some cases even deliberately provoke them. We should not forget that wild cards can also be beneficial events, whose potential we want to be able to exploit.

Some use wild cards as a synonym for weak signals. Hiltunen [1, p. 96] however, defines wild cards as events with a huge impact whereas weak signals are signs of events or emerging issues, such as wild cards. Whether or not one consider these two terms as synonyms, depends how one defines weak signals. If one accepts that weak signals can be both events and signs of events, then wild cards and weak signals can be used interchangeably. However, if one thinks that weak signals are not events themselves but indications of them, wild cards and weak signals are not synonyms. Instead, it can be perceived that weak signals precede wild cards. Thus, weak signals can be employed as a means to anticipate wild cards $[1,20]$.

Wild cards are often confused with gradual change [1]. Gradual change, like the change from dry toilet to water closet, had a significant impact, but it was not rapid as it was possible to observe the change and adapt to it. In the case of wild cards, there is only little time to react before it takes place. An example of wild card could be the Nokia water crisis described in the introduction. There is some disagreement about whether a wild card can be anticipated at all. Petersen and Steinmüller [18] distinguish three types of wild cards: 1) events that are known and relatively certain to occur but without any certainty as to timing (e.g. the next earthquake), 2) future events that are unknown to the general public (or even the professionals) but that could be discovered if we only consulted the right experts or if we had adequate models (e.g. impacts of climate change), and 3) intrinsically unknowable future events that no expert has in mind, where we lack concepts and means of observation (the unknown unknowns). The last type of wild cards, the unknown unknowns we can only judge by hindsight. Category two wild cards are the ones that we could possibly anticipate and are of interest here.

Mendonça et al. [20] argue that it is sometimes possible to anticipate wild cards in advance as weak signals of it are available (see also [1]). The question is more about if someone notices these signals and is able to make "correct" interpretations on it. In the case of Nokia water crisis the weak signals were observations by consumers on the weird appearance of water. Consumers complained about these to the water works, but these weak signals were not taken seriously. It was assumed that foaming of the tap water and its weird smell and taste were due to pressure changes in the water distribution network. It was only after two days when people were reporting stomach problems that the issue was taken seriously. ${ }^{2}$ If the signals, concerns of people, had been taken seriously and reacted earlier on, it would have been possible to limit the extent of the water epidemic.

\subsection{Methodology for Identifying and Analysing Weak Signals and Wild Cards}

Some methods to analyse weak signals have been developed and can be found in literature. E.g. Ansoff has

\footnotetext{
${ }^{2}$ For a detailed description of the Nokia Water Crisis (in Finnish) see Seeck et al. [21].
}

created a Weak Signal Issue Management System (Weak Signal SIM). Hiltunen [1], however, argues that the practical use of this approach appears to be very mechanistic as there is no space for creativity and intuition.

According to Schultz [22], a basic approach to scan environment for weak signals consists of the following phases: 1) choosing from five to nine information sources, that should preferably be from different sectors and should cover both, specialist and fringe sources, 2) creating a scanning database, including the title, source, description and implications of the signal, 3) evaluating scan "hits", are they subjectively or objectively new, are they confirming, reinforcing or negating, and 5) looking for interdependencies, feedback delays and repeating patterns in the scanned data (see also Linturi [23]).

Linturi [23] has developed an online methodology Signalix to hunt down weak signals. Signalix is a comprehensive process consisting of altogether 12 phases. Summarising, this method starts by choosing a problem and describing it (phase 1 ), next the context of problem is analysed (e.g. analysing the system, trends, values and memes related to the problem; phases 2-6), third part is the actual search for signals, i.e. looking for signals of the chosen meme in sources (phase 7), signals are then analysed by classifying them into strong (trends) and weak and then assessing the signals using e.g. Delfoi (phases 8-9). In the last phases (10-12) the analysed signals are used to create scenarios and a vision, and a system to monitor development systematically.

As weak signals are seen to precede or indicate wild cards, identifying weak signals and interpreting them can produce information on wild cards. Mendonça et al. [20] can be seen to favour this type of approach. Another possibility is to directly try to identify wild cards and then assess and monitor them by identifying weak signals that could indicate these wild cards. For example, Petersen and Steinmüller [18] introduce wild cards methodology that starts by identifying wild cards. This can be done by using published lists of wild cards. However, they recommend collecting or inventing wild cards specific to case in question. In the next phase, the identified wild cards are assessed and their amount is narrowed down so that only the ones considered most relevant will be considered in the following phases. The third phase consists of monitoring weak signals of the wild card. Fourth, options for action (to prevent, to prepare for, or to promote wild cards) are discussed.

The method we used in this study does not directly follow any of the ones described above. Most often weak signal methods have been created for organisations to aid them in anticipating futures, scenario processes and creating their strategies. Our case, however, was quite different. We were not looking to create scenarios or strategies for the whole field, but instead trying to look for signals that the actors in the field could possibly use in their strategy processes. Thus, the methodology that was chosen was quite loose and in a sense, it could be called data-driven. Our choice is supported by Rossel [16, p.318], who argues that weak signal analysis should not be a one-size-fits-all approach, but that the characteristics of the organisation or object of study should influence the way analysis is conducted.

Similarily to Linturi's [23] Signalix, we started by discussing the possible problems and issues related to water services. Following Coffman [24] we were discussing about things that just feel funny about our case and things that we see as happening, but cannot really pin them down. In a sense, this 
phase resembles also the first phase of wild cards methodology: inventing things that could happen [18, p. 4-5]. Next, we decided on the sources or the material that we will go through to look for weak signals and started scanning these.

\subsection{Materials}

Hiltunen [25] has made a survey study asking futurists what they considered to be good sources of weak signals. In her results, personal connections were empathised and overall favoured sources were scientists/researchers, futurists, colleagues, academic and scientific journals, and reports of research institutes. As was discussed in the introduction, the water sector is rather conservative. Thus, we felt that it would not be that beneficial to use field experts or their writings as a source. Instead, we chose as our material Finnish newspaper and magazine articles that were not related to water sector directly. The idea was that these materials could indicate weak signals of the wider societal change. In addition, we used some other materials, like blog texts. These were used in the first phase when we were trying to scan for interesting issues and problems from outside the water sector that could be used as analogies in the sector. Hiltunen [25] also recommends the use of so called peripheral sources (such as arts, science fiction, alternative press, blogs).

We decided to cover a time span of one year of the newspapers and magazines (March 2010 - March 2011). The newspapers chosen were Aamulehti and Lapin Kansa. We did not read all papers published during the particular year, but focused on papers of $11^{\text {th }}$ and $27^{\text {th }}$ day of each month. Aamulehti is the newspaper of Tampere area and Lapin Kansa is published in northern Finland. These papers were chosen to get a wider geographical view. Magazines chosen were Image and Kuluttaja. Image magazine covers a wide range of phenomena from popular culture to politics, and it can be characterized as a trendsetter. We chose Image as we thought it might offer some fresh perspectives. Kuluttaja magazine is published by the Finnish Consumer Agency and it focuses on reviews of products and services. Kuluttaja was chosen to give perspectives of changes in consumer culture and preferences of people.

\section{Results}

As described, we started the empirical part of this study by discussing things that feel funny about water services and things that are happening, but we cannot really pin them down. Second, we read the newspapers and magazines. The results are shortly described in this section.

\subsection{Inventing Things That Could Happen}

First, we discussed some emerging issues in the water services sector. Basically, these were issues that had puzzled us. One such issue is bottled water. Many water sector experts condemn bottled water as a totally useless, ridiculous and stupid product and downplay people's reasons to choose bottler water over tap water. The markets for bottled water, however, have been rapidly growing so consumers are appealed to it. Then again, the biggest challenge in Finnish water services sector is the aging infrastructure and the growing renovation debt [10]. If the water utilities cannot keep up with the needed infrastructure renovation pace because of lack of required resources, the quality of tap water will be endangered at some point. Could one option be to accept lowered quality of tap water and use bottled water for drinking and other purposes requiring higher quality of water? After all, about $95 \%$ of tap water is used for other than drinking and cooking purposes, such as flushing the toilets.

Another issue discussed was water-related crisis - will there be another crisis like the one in Nokia in 2007? What could trigger such a crisis? Could one cause be the use of various plastic compounds in the distribution networks, such as new epoxy plastics in renovation? There is no experience of these materials in the long run. There have, however, been some worries related to bottled water that some more or less hazardous chemicals would be released from the plastic bottles into water (e.g. [26]). Could plastic water pipes jeopardize people's health in the future? Why would plastic be harmful only as bottle material but not as pipe material? Also, what if it is found out that the plastic pipes are not durable in use and need to be replaced only after few years in use? This would not necessarily compromise people's health directly, but would be a huge financial burden to the water services providers and, after all, to the customers.

Third issue was the role of the customer in water services. In Finland, water services provision is the responsibility of municipalities. Is there a tension between the roles of a customer and citizen? Will people trust public services in the future? Will the requirements of people significantly change in the future? Quite many water services experts seem to think that many challenges of the field could be resolved by moving water services further away from political decision-making, i.e. especially that water works would be financially separate from the municipalities. How will this impact water services and people's perception of these services?

\subsection{Scanning the Newspapers and Magazines}

One issue that seemed to rise from scanning the newspaper and magazine articles were customers' changing expectations. Both public and private services need to be convenient and readily available. For example, in Image 9/2010 the chief editor claims that oranges are too inconvenient for modern consumers as peeling them is too laborious. Then again, in Aamulehti 11.11.2010 it is written that older generations are used to being passive objects of services. Younger generations, instead, require more and want to make individual choices about service that will take into consideration their personal values and needs. There was also quite many articles about how we should focus more on the ultimate purpose of services and think about what people actually expect of the services (these were especially about the health services e.g. Image 3/2011; Aamulehti 27.3.2011; but also about the construction sector Aamulehti 11.11.2010 and business, e.g. about Nokia forgetting its customers Aamulehti 11.9.2010). In Lapin Kansa 27.4.2010 it was demanded that an individual and his or her needs should be the starting point of different public services, not the way these services are organized or minimizing their costs.

Another issue is the apprehension about chemicals, or even "chemophobia". This was especially visible in the Kuluttaja magazines reviews about consumer products (e.g. in Kuluttaja $3 / 2010$ prams were tested for hazardous chemicals). People also expect more open information about chemical consistency and additives of different products in an understandable way (e.g. Kuluttaja 8/2010 and Kuluttaja 4/2010). It seemed that some businesses had already responded to these demands. An example of this is a full-page advertisement by a dairy 
producer Arla-Ingman on their new margarine Arla Ingmariini that is "totally without additives" (Aamulehti 11.3.2011).

A third issue that we found interesting was related to political decision-making. First of all, there were some articles about how power has been shifted away from democratically chosen political decision-makers to professional civil servants (e.g Image 3/2011). For example, in Image 8/2010 the victory of an Icelandic political party that was set-up just as a joke was explained by the frustration of people in the toothless political decision-making in the past. Politicians do not want to make hard decision or discuss values. Aamulehti 11.11.2011 also quoted newspaper Karjalainen that it is about time that the politicians take power and carry their responsibility, they should not anymore hide behind civil servants. There were quite a few articles that called for more open, transparent and participatory decision-making processes. E.g. in Aamulehti 27.11.2010 there was an article about how the language used by civil servants is incomprehensible to lay people making it impossible for them to follow-up decision-making and take part in it.

There were also some examples of how decision-making processes had been improved. For example, Lapin Kansa 27.9.2010 wrote of the civil servants responsible for urban planning and waste management in the town of Rovaniemi, Finland who participate in open coffee meetings with local residents. In these unofficial gatherings people feel easier to ask these civil servants about issues puzzling them, and then again share their personal views. Another issue, quite expectedly, was about utilizing internet and social media in public services. Quite a surprising news, however, was the extent that the Finnish tax authorities are already utilizing blogs and discussion forums in their work (Lapin Kansa 11.7.2010).

These are just a few examples of the news that were chosen and analyzed. In the next section, these findings are assessed against theories on weak signals and wild cards.

\section{Discussion and Assessment of the Results}

We will start by assessing our findings introduced in previous section. One problem here is, as Hiltunen [1] points out, that the actual value or worth of weak signals can only be judged with hindsight. The same can be said to apply also to wild cards. We scanned for the weak signals and wild cards in the spring 2011. Now, after more than three years we can say that there is no clear indication that customers' changing expectations or changing political processes would have had a major implications for the water sector, at least yet. Then again, the apprehension about chemicals seems to be a trending topic in the water sector. The Finnish Ministry of Social Affairs and Health together with the Ministry of Environment stated in their press release that certain plastic water pipes had been found to cause taste and odour problems with tap water [27]. This was reported also in the news and raised some discussion in social media (e.g. blogs). Still, we cannot know if our interpretations are correct or useful to the water services sector. Furthermore, as Rossel [5] argues, even based on hindsight, we cannot rule out false positives (discovering later on that the evidence misleading) and false negatives (being right but on false grounds).

Thus, we will try to assess our findings against theories on weak signals and wild cards. First of all, the reliability of findings depends on the resources or materials used. According to Hiltunen [1, p. 104] weak signals can be divided into primary and secondary exosignals. Primary signals are directly connected to an emerging issue and are, for example, visual observations of the issue. When primary signals are interpreted and presented e.g. in newspapers, they turn into secondary signals. Hiltunen [1] warns that there is a risk with secondary signals being distorted or even fictious. Probably one of the biggest weaknesses in this study was the material chosen. We used mainly newspapers and magazines, and thus we relied on secondary signals.

A further weakness with the materials used in systematic scanning was that none of them was really peripheral or alternative as is recommended [25] but they represented mainstream. In the background, identifying the emergent issues, we did use blog writings but these were not used in the systemic scanning. The original idea was to include also peripheral sources for scanning, but this was given up due to lack of time.

As some of the futurists in Hiltunen's [25] study emphasised, it is not the sources of weak signals that are important, but rather processing of them. Thus, the actual process will be discussed next. As described in methodology section, we started by discussing issues that had puzzled us. According to van der Heijden [28], people have tacit knowledge consisting of isolated observations and experience that they have not yet been able to integrate with their codified knowledge and this is also why they do not understand meaning of these very clearly. Furthermore, he describes weak signals as this sort of unconnected insights and knowledge. It could be said that our first discussions on the problems and issues was an attempt to try to understand the weak signals we had encountered earlier on. Now, afterwards trying to separate the signals from the interpretations of the emerging issues they are signalling is quite impossible and would be artificial.

Similarly, if one looks at the findings described in the previous section, they are not descriptions of the signals themselves but more interpretations. This is related to the debate on whether weak signals can be objective or not and whether the signal can be separated from its interpretation. It was obvious in the process, that nearly all of the signals we found were somehow related to the problems we had discussed earlier on. One could accuse us of scanning for signals that further strengthen our previous understanding. Hiltunen [1 p, 105] describes this as "collective blindness", when only signals that strengthen a vision are allowed inside an organization. She argues that this can happen easily with secondary exosignals. However, starting with a problem or issue in mind, is also part of the many methodologies described in the literature (see e.g. Petersen \& Steinmüller [18]; Linturi [23]).

Interpretation, in our case, was mainly based on assessing the relevance of signals in water services sector. Hiltunen $[1$, appendix 3] warns of emphasising the relevance too much as it can cause filtering especially of signals that are inconvenient but could have a big impact in the future. Then again, van der Heijden [28] argues that weak signals reach our consciousness because we intuit that they have some relevance to our situation (see also [4]). It is a tempting idea that we could scan our environment without preconceptions. However, in practice we do not think that this is possible. Instead, we agree with Rossel [16, p. 316] who argues that we should make "our assumptions as explicit as possible, and part of the weak signal identification process itself (i.e. taking into account the 
different usages we have of weak signals, according to our diverse roles and contextual interests)". The first part of the results, describing the issues that puzzled us, is also an attempt to make our assumptions explicit.

Another issue that could probably have improved the results by helping to avoid the filtering (e.g. past experiences, educational background, political interests [4]) to some extent would have been to include more people in the process with more diverse backgrounds. If their preconceptions, assumptions and tacit knowledge is diverse this would enhance the diversity of the results. The futurists in Hiltunen's [25, p. 22] study emphasized interaction, openness and discussion in finding weak signals. Or as Mendonça et al. [4] state, the process is actually structured networked communication. In addition, it would be important to pay more attention the design of the frame of interpretation (see also Jørgensen [29]).

One could criticize our approach as being too loose. Moijanen [15, p. 57] for example argues that scanning for weak signals requires systematic search as one must be able to distinguish weak signals from the background noise. Also Holopainen and Toivonen [3] criticise the loose use of the concept of weak signals as it blurs the identification of the really relevant and strategic changes. However as Mendonça et al. remind [4] the distinction between noise and signal is not so straightforward but it depends on the interpretive context.

Furthermore, in our view, a too mechanistic and rigid approach would probably limit the findings and kill the creativity of the process. The whole idea, after all, is to try to break mental models and come up with events that you would not have thought about otherwise. It can also be argued that a more loose approach does not so easily create false sense of security (see e.g [9]). A very rigid and heavy approach, on the other hand, can create the illusion that the operational environment is under control and weak signals and wild cards are being managed. Mendonça et al. [4] argue that thinking about weak signals can actually help to acknowledge the limits of foresight abilities.

Our interpretations and analysis were mostly based on analogies, i.e. scanning for signals from other sectors and analyzing what these could mean in the water services sector. This kind of approach is criticized by van der Heijden [28]. He argues that the validity of such analogies cannot be assessed and thus, it must be concluded that the resulting subjective probabilities are untestable, arbitrary and meaningless. However, Ruuttas-Küttim [in 10] encourages combining different contexts to weak signals in order to see their real potential.

Furthermore, it is questionable whether our findings really count as weak signals or wild cards. For example, the issue with the possible chemical contamination of tap water from the plastic pipes is more likely a gradual change than a wild card, as it is not a rapid development and water works could monitor this and change their behaviour in case some concerning results would appear. Most of the other issues we discussed are also more gradual than rapid in nature. Thus, it could be said that we were not able to recognise actual wild cards. However, it needs to be remembered that it is also important to monitor weak signals for gradual change. As Hiltunen [19] points out, people tend to ignore weak signals indicating gradual change.

Moreover, one could say that our weak signals are not signals but more like trends. This is due to the fact that they are presented in a combination with the interpretations and they have been clustered together. Hiltunen [1] actually reminds that single weak signals do not tell us much about futures, but a number of weak signals might tell us something about emerging trends in the future. She argues, thus, that weak signals should be clustered to trends. One of the trends identified in our study could be the customers' growing demands and willingness to make own choices. In the water services sector, it might be useful to think about how the service could be made more customer-oriented. One should also think about what the ultimate purpose of the service is. This is also related to trend of more open, political and transparent decision-making. If politicians would assume more power and responsibility over their decisions, how would this impact water services sector? Would the resources to provide safe service be better or worse? What if customers and citizens would be better aware of also decision-making related to water services? Would this increase or decrease resources? These are only very preliminary thoughts and it would be the next phase that we should focus on developing these trends further and even create scenarios based on them.

It is debatable, whether the signals we found could be considered as weak. This again is related to our choice of material and methodology. According to Hiltunen [1], a key characteristic of weak signal is their low visibility, as it usually appears only in a single channel and locally. Our sources, the newspapers and magazines, however are quite widely read and their visibility is not that limited. Another criteria proposed to describe the "weakness" of signals refers to the inability to give meaning to them $[28$, p. 8$]$. In comparison, "strong" signals would be such that we can clearly understand the potential implication. Based on this definition our findings could be characterised as weak signals. They can be considered to be strong signals in their original context, but when they are transferred to the context of water services sector their implications are not clear and thus, they can be said to represent weak signals.

Hiltunen's [1] informal test of weak signals, based on the reactions of colleagues, seems to support the claim that our findings would be weak signals. According to Hiltunen, if your colleagues oppose a signal or it is not really talked about (taboo) it can be considered weak. We presented our colleagues the idea of bottled water replacing drinking water. First, there was a long silence which was followed by declarations of the stupidity of the whole idea. It seemed that they were upset by the sheer idea of taking bottled water seriously. This reinforced our idea that it would be important to expose our sector to thinking in new ways and breaking conservative mental models to better prepare, prevent or take advantage of these events in the future. According to Holopainen and Toivonen [3] Hiltunen's criteria is highly subjective. Once again, this reverts to the discussion on the objectivity and subjectivity of the weak signals, and the problems of assessing signals beforehand. In the end, we argue that the "rightness" or "correctness" of the weak signals and wild cards is secondary and the primary importance is in the process itself. Studying theories on weak signals and wild cards, scanning the papers for them and analysing our findings helped us to attune our senses to signals from outside the water sector and to acknowledge the limits of knowing about the future. Following Mendonça et al. [4], we maintain that the value lies in the potential for social and organisational learning: building capability to deal with uncertainties, the unknowns and the unexpected. This is an ongoing process and "preparing for future unknowns is always an unfinished business" [4, p. 223]. 


\section{Conclusions and Recommendations}

As was discussed, it is debatable whether any of the "signals" we discovered were really weak signals by scientific definition. Similarly, we were not able to identify wild cards. Furthermore, our approach did not follow guidelines strictly. We cannot yet show that our findings would be "correct" as weak signals and wild cards can only be judged with hindsight. However, we do not think that this exercise was useless but quite the contrary. We have presented some of our findings to other people on water services sector. Reactions have ranged from ignorance to anger. We argue that scanning for weak signals and wild cards can help one to step out of one's comfort zone and think also about the inconvenient issues. Even if the weak signals and wild cards would not materialize in the future, it is useful to challenge oneself to think differently. Based on our experience, we argue that the potential of the weak signals and wild cards approach lies in the practical application, not in scientific strict methodology and rigid interpretations of weak signals and wild cards.

Sustainability of water services is one key issue for the well-being of people and functioning of societies. As the world changes into more complex, unpredictable and uncertain, this will eventually impact also the water services sector increasingly. As the sector is static and conservative, it is not very agile reacting to changes. This makes the need for strategic thinking and planning even more evident. The idea of weak signals and wild cards can play an important part in this.

Water services, by their nature, strive for keeping balance; a lot of effort is put to ensuring that nothing unwanted occurs. In this regard it is a sort of crisis management long before any crisis takes place. Thus, as Edwards [30] argues, vigilant organisations having a capability of social learning success well in avoiding crises and accidents, even if their operations are heavily based on technologies. More theoretically speaking, exploring weak signals and wild cards can serve as a communication and reflection practice in which new information is processed, thereby producing and decreasing entropy in the social system. Organisation's ability to continuously produce and decrease entropy can ensure its autopoietic process by which an organisation can evolve over time [31]. We therefore see that weak signals and wild cards are useful methods for improving vigilance and learning in the organisations of the water services sector. All in all, we recommend that water utilities and other central actors in the field of water services to apply a loose approach on weak signals and wild cards as a part of their organisational culture. We see this more as an ongoing activity and future-oriented organisational philosophy rather than a strict scientific method. For example, in its simplest form this could be a coffee table discussion covering daily newspapers and some social media sources, reflecting what these could mean for the water services and trying to identify relations between the potentially meaningful signals. This would help to build a dynamic, learning organisation that is open to sense the wider society around it.

\section{Acknowledgments}

Financial support from the Alfred Kordelin Foundation and the Foundation for Municipal Development is gratefully acknowledged.

\section{References}

[1] Hiltunen E: Weak Signals in Organizational Futures Learning. Doctoral Dissertation. Helsinki, Helsinki School of Economics, 2010.

[2] Miller R, Rossel P, Jørgensen U. Future studies and weak signals: A critical survey. Futures 2012;44: 195-197.

[3] Holopainen M, Toivonen M. Weak signals: Ansoff today. Futures 2012; 44: 198-205.

[4] Mendonça S, Cardoso G, Caraça J. The strategic strength of weak signal analysis. Futures 2012;44: 218-228.

[5] Rossel P. Early detection, warnings, weak signals and seeds of change: A turbulent domain of futures studies. Futures 2012;44: 229-239.

[6] Saritas O, Smith JE. The Big Picture - trends, drivers, wild cards, discontinuities and weak signals. Futures 2011;43: 292-312.

[7] Nafday AM. Strategies for Managing the Consequences of Black Swan Events. Leadership and Management in Engineering 2009; 9: 191-197.

[8] Kahneman D: Thinking, fast and slow. London: Penguin Books, 2011.

[9] Taleb NN: The Black Swan: The Impact of the Highly Improbable. New York: Random House, 2010.

[10] Heino OA, Takala AJ, Katko TS. Challenges to Finnish water and wastewater services in the next 20-30 years. EWater 2011; 1:1-20.

[11] Dominguez D: Handling future uncertainty - Strategic planning for the infrastructure sector. Doctoral Dissertation. Zurich: ETH, 2008.

[12] Meadows DH: Thinking in Systems: A Primer. Vermont: Chelsea Green Publishing, 2008.

[13] Swyngedouw E: Social Power and the Urbanization of Water. Flows of Power. Oxford: Oxford University Press, 2004.

[14] Castro JE. Systemic Conditions and Public Policy in the Water and Sanitation Sector. In: Castro JE, Heller L (Eds), Water and Sanitation Services, Public Policy and Management. Earthscan, 2009, pp. 19-37.

[15] Moijanen M: Heikot signaalit tulevaisuudentutkimuksessa [Weak signals in futures research]. Futura, 2003;4: 43-60.

[16] Rossel P. Weak signals as a flexible framing space for enhanced management and decision-making. Technology Analysis \& Strategic Management, 2009; 21: 307-320.

[17] Hiltunen E. The future sign and its three dimensions. Futures 2008;40: 247-260.

[18] Petersen JL, Steinmüller K. Wild Cards. In: Glenn JC, Gordon TJ (Eds.) Futures Research Methodology. Version 3.0. The Millennium Project, 2009.

[19] Hiltunen E. Was it a Wild Card or Just Our Blindness to Gradual Change? Journal of Future Studies 2006; 11: 6174.

[20] Mendonça S, Pina e Cunha M, Kaivo-oja J, Ruff F. Wild cards, weak signals and organisational improvisation. Futures 2004; 36: 201-218

[21] Seeck H, Lavento H, Hakala S: Kriisijohtaminen ja viestintä. Tapaus Nokian vesikriisi [Crisis management and communications. The case of Nokia water crisis.] Helsinki: The Association of Finnish Local and Regional Authorities, 2008.

[22] Schultz WL. Environmental Scanning: A Holistic Approach to Identifying and Assessing Weak Signals of Change. Retrieved on 16 May 2011 from http://www.infinitefutures.com/essays/prez/holescan/sld00 1.htm

[23] Linturi H. Heikkoja signaaleja metsästämässä [Hunting for weak signals]. Retrieved on 15 May 2011 from 
http://www.futunet.org/en/materiaalit/metodit/2 metodit/3 _signalix?C:D=347697\&selres $=347697$

[24] Coffman BS. Weak Signal Research. Part I: Introduction. Retrieved on 15 May 2011 from http://www.mgtaylor.com/mgtaylor/jotm/winter97/wsrintr o.htm

[25] Hiltunen E. Good Sources of Weak Signals: A Global Study of Where Futurists Look For Weak Signals. Journal of Futures Studies 2008;12: 21-44.

[26] Westerhoff P, Prapaipong P, Shock E, Hillaireau A. Antimony leaching from polyethylene terephthalate (PET) plastic used for bottled drinking water. Water Research 2008;42: 551-556.

[27] The Finnish Ministry of Social Affairs and Health. Uusista muovisista vesijohdoista aiheutunut paikoin haju- ja makuhaittoja talousveteen [New plastic pipes have caused some odour and taste problems in tap water]. Retrieved 9 July 2014, from http://www.stm.fi/tiedotteet/tiedote//view/1887128

[28] van der Heijde K. Scenarios, Strategy and the Strategy Process. Presearch 1997; 1, 1-32.

[29] Jørgensen U. Design junctions: Spaces and situations that frame weak signals - the example of hygiene and hospital planning. Futures 2012: 44: 240-247.

[30] Edwards P.N. Infrastructure and Modernity: Force, Time, and Social Organization in the History of Sociotechnical Systems. In: Misa TJ, Brey P, Feenberg A (Eds.). Modernity and Technology. Cambridge, MA: MIT Press, 2003, pp. 185-226.

[31] Maturana HR, Varela FJ. Autopoiesis and Cognition: The Realization of the Living. Dordrecht, Holland: D. Reidel Publishing Company, 1980. 